Artículo Original. Mayo-Agosto 2017; 7(2):14-21. Recibido: 17/02/2017 Aceptado: 06/04/2017.

http://dx.doi.org/10.21929/abavet2017.72.1

\title{
Uso de extracto enzimático de Pleurotus ostreatus sobre los parámetros productivos de cabras
}

Use of enzymatic extract of Pleurotus ostreatus on production parameters of goats

\author{
Trejo-López Teresa teretrejolopez.m.c@gmail.com, Zepeda-Bastida Armando \\ azepeda@uaeh.edu.mx, Franco-Fernández Jesús mfranco@uaeh.edu.mx, Soto-Simental \\ Sergio sotos@uaeh.edu.mx, Ojeda-Ramírez Deyanira dojeda@uaeh.edu.mx, Ayala- \\ Martínez Maricela*ayalam@uaeh.edu.mx
}

Instituto de Ciencias Agropecuarias, Universidad Autónoma del Estado de Hidalgo. *Autor responsable y de correspondencia: Ayala-Martínez Maricela, Instituto de Ciencias Agropecuarias, Universidad Autónoma del Estado de Hidalgo. Ave. Universidad s/n km 1. Tulancingo, Hidalgo.México.

\section{RESUMEN}

La utilización de enzimas fibrolíticas exógenas comerciales (celulasas y xilanasas) ha sido ampliamente empleada en producción animal, con la finalidad de complementar la actividad de las enzimas endógenas, para lograr un incremento en la respuesta de producción de leche. En esta investigación se pretende utilizar como alternativa el uso de enzimas exógenas obtenidas del sustrato gastado de Pleurotus ostreatus con el fin de evaluar los parámetros productivos de cabras. Los resultados mostraron que la inclusión en la dieta de este extracto enzimático, tuvo efecto sobre la ganancia diaria de peso, producción de leche y consumo de alimento. Lo que sugiere que el extracto enzimático obtenido del sustrato gastado de Pleurotus ostreatus, puede ser una alternativa para mejorar los parámetros productivos de las cabras.

Palabras clave: leche, extracto enzimático, cabras.

\begin{abstract}
The use of commercial exogenous fibrolytic enzymes (cellulases and xylanases) has been widely used in animal production, in order to complement the activity of the endogenous enzymes, to achieve an increase in milk production response. In this research, we intend to utilize as an alternative the use of exogenous enzymes obtained from the spent substrate of Pleurotus ostreatus in order to evaluate the productive parameters of goats. The results showed that the inclusion in the diet of this enzymatic extract had an effect on daily weight gain, milk production, and consumption. This suggests that the enzymatic extract obtained from the spent substrate of Pleurotus ostreatus may be an alternative to improve the productive parameters of goats.
\end{abstract}

Keywords: milk, extract enzymatic, goat.

\section{INTRODUCCIÓN}

Para obtener mejor producción en rumiantes es importante considerar el tipo de forraje que es consumido por estos, debido a que aunque cuentan con una gran variedad de enzimas fibrolíticas en el rumen (celulasas, xilanasas, B-glucanasas, pectinasas), que se encargan de intervenir en el proceso de degradación del material alimenticio; la presencia de compuestos indigestibles como la lignina, demeritan el aprovechamiento de los forrajes y con ello la producción de estos animales (González, 2002), tal es el caso de las 
cabras producidas principalmente en zonas áridas y semiáridas, que en épocas de sequía consumen forrajes con poca digestibilidad, debido a la alta cantidad de lignina (Salinas et al., 2015); por tal motivo, la utilización de enzimas fibrolíticas exógenas comerciales (celulasas y xilanasas) han sido ampliamente empleadas con la finalidad de complementar la actividad de las enzimas endógenas, demostrando un incremento en la respuesta de producción de leche (Beauchemin et al., 2000; González, 2002), además de mejorar la ganancia de peso y condición corporal (Caja et al., 2003).

Para obtener enzimas exógenas se han buscado diferentes alternativas que sean amigables con el medio ambiente y con la economía, como la propuesta por Sánchez et al., (2015), quienes utilizaron sustrato gastado de Pleurotus ostreatus, ya que produce enzimas fibrolíticas que pueden degradar materiales lignocelulósicos, debido al complejo enzimático que forman (celulasas, xilanasas, ligninoperoxidasa (Lip), manganeso peroxidasa (MnP), lacasas y peroxidasa versátil (VP) (Sánchez et al., 2015), con la finalidad de incrementar la disponibilidad de celulosa y hemicelulosa para los microorganismos ruminales (Van Kuijk et al., 2015).

El objetivo del presente estudio fue evaluar los parámetros productivos de cabras alimentadas con extracto enzimático de sustrato gastado de Pleurotus ostreatus.

\section{MATERIAL Y MÉTODOS}

El extracto enzimático se obtuvo del sustrato gastado de Pleurotus ostreatus, el cual fue homogenizado y muestreado por cuarteo, hasta obtener $250 \mathrm{~g}$, para ser mezclado con $500 \mathrm{~mL}$ de agua destilada; posteriormente se agitó vigorosamente, se filtró y la parte líquida se concentró en un rotavapor a $80^{\circ} \mathrm{C}, 75 \mathrm{rpm}$, durante $1 \mathrm{~h}$. Se evaluó la cantidad de proteína soluble (Bradford, 1976) contenida en el extracto enzimático, actividad enzimática de celulasa y xilanasa por la técnica de azúcares reductores, con el método del ácido dinitrosalisílico (Miller, 1959) modificado por Loera y Córdova (2003) y Márquez et al., (2007) y lacasas por sistema de reacción de ABTS (2, 2' Azino-bis 3-etilbenzenothiazolina-6-ácido sulfónico) (Bourbonnais et al., 1997; Márquez et al., 2007).

Se incluyeron $50 \mathrm{~mL}$ de extracto enzimático (Cuadro 1 y 2) en $200 \mathrm{~g}$ de concentrado isoproteico (20\% PC), isoenergético (2.6 Mcal kgMS-1) e isofibroso (14.7\% FDN, $8.4 \%$ FDA y $1.7 \%$ Lignina); para conformar cuatro tratamientos $(0,0.625,1.25$ y $2.5 \mathrm{~g}$ de extracto enzimático seco). Se utilizaron 24 cabras distribuidas completamente al azar en cuatro tratamientos, el concentrado fue ofrecido durante la ordeña diariamente, antes de que salieran a pastoreo, durante 15 d de adaptación y 45 d de experimentación. Se midió consumo $\mathrm{d}^{-1}$, ganancia diaria de peso (GDP) y producción de leche $\mathrm{d}^{-1}$. 


\section{ANÁLISIS ESTADÍSTICO}

Los datos obtenidos tuvieron un arreglo completamente al azar y se analizaron por medio de un procedimiento GLM; para la comparación de medias se utilizó una prueba de Tukey con una probabilidad de $\mathrm{P}<0.05$, con el programa estadístico IBM SPSS 2011 (SPSS, 2011) y Sigma Plot 10 (Sigmaplot, 2006).

\section{RESULTADOS Y DISCUSIÓN}

El extracto enzimático de sustrato gastado de Pleurotus ostreatus presentó mayor cantidad de proteína soluble, actividad enzimática de xilananas y celulasas (Cuadro 1) a lo reportado por Ayala et al., (2011), en un extracto enzimático obtenido de sustrato gastado de Agaricus bisporus a diferentes días de cultivo, de manera contraria la actividad de lacasa (Cuadro 2). Sin embargo, Arce et al., (2013a) obtuvieron valores inferiores de xilanasas, celulasas y lacasas, al evaluar el extracto de enzimas lignocelulolíticas de un basidiomiceto, Fomes sp. EUM1. Por otro lado, Márquez et al., (2007) quienes realizaron un estudio de Pleurotus ostreatus IE8 a diferentes días de fermentación, reportaron una actividad enzimática inferior a lo obtenido en esta investigación.

\begin{tabular}{cc}
\hline Parámetro & Ul MS g $^{-1}$ \\
\hline Xilanasas $^{1}$ & $32227.72 \pm 1565.06$ \\
Celulasas $^{1}$ & $69.03 \pm 48.59$ \\
Proteína $^{2}$ & $10307.42 \pm 3700.45$
\end{tabular}

${ }^{1}=$ Unidad internacional (UI) para xilanasas y celulasas como la cantidad de enzima que libera $1 \mu \mathrm{mol}$ de azúcar reductor (expresado como equivalentes de xilosa y glucosa) por min.

${ }^{2}=\mu \mathrm{g} \mathrm{MS} \mathrm{g}^{-1}=$ Microgramo por $\mathrm{g}$ de materia seca

UI $\mathrm{MS} \mathrm{g}^{-1}=$ Unidades internacionales por gramo de materia seca

Cuadro 1. Proteína soluble, actividad enzimática de xilanasas y celulasas de extracto enzimático de sustrato gastado de Pleurotus ostreatus.

\begin{tabular}{cc}
\hline Semana & \multicolumn{1}{c}{ UI MS g $^{-1}$} \\
\hline $\mathbf{1}$ & $4535.41 \pm 1040.03^{\mathrm{a}}$ \\
$\mathbf{2}$ & $1868.05 \pm 408.55^{\mathrm{b}}$ \\
$\mathbf{3}$ & $2684.02 \pm 584.34^{\mathrm{ab}}$ \\
$\mathbf{4}$ & $3029.16 \pm 273.02^{\mathrm{ab}}$ \\
$\mathbf{5}$ & $2450.69 \pm 472.38^{\mathrm{ab}}$ \\
$\mathbf{6}$ & $2366.66 \pm 223.91^{\mathrm{ab}}$
\end{tabular}

$\mathrm{a}, \mathrm{b}=$ Literales indican diferencia estadísticamente significativa $(\mathrm{P}<0.05)$. Unidad internacional (UI) definida como la cantidad de enzima que produce $1 \mu \mathrm{mol}$ de substrato oxidado por min.

UI $\mathrm{MS} \mathrm{g}^{-1}=$ Se considera una unidad internacional por gramo Media \pm error estándar.

Cuadro 2. Actividad enzimática de lacasas obtenidas del extracto enzimático del sustrato gastado de Pleurotus ostreatus. 
Considerando que el peso inicial y el peso final de las cabras (Cuadro 3) no fueron diferentes ( $P>0.05)$, el extracto enzimático de sustrato gastado de Pleurotus ostreatus aumentó $(\mathrm{P}<0.05)$ la ganancia diaria de peso a partir de la semana cuatro (Cuadro 4), lo cual coincidió con lo reportado por González (2002), quien obtuvo un incremento del peso vivo de cabras en lactación al incluir en su alimentación enzimas comerciales Promote®. Al igual que lo obtenido por Bueno et al., (2013), quienes ofrecieron altas dosis de enzimas fibrolíticas (Fibrozyme ${ }^{\circledR}$ ) a corderos alimentados con paja de avena, Arce et al., (2013b) quienes ofrecieron extracto lignocelulótico de basidiomiceto Fomes sp. EUM1, el cual fue esparcido sobre el alimento de corderos, encontrando mejoras sobre la ganancia diaria de peso de los corderos y Torres et al., (2013), quienes evaluaron enzimas fibrolíticas de Trichoderma longibrachiatum, Fomes fomentarius y Cellulomonas flavigena, sobre el crecimiento de corderos utilizando $60 \%$ de forraje.

\begin{tabular}{cccc}
\hline Tratamiento & Peso inicial & $\begin{array}{c}\text { Peso final } \\
\mathrm{kg}\end{array}$ & Ganancia de peso total \\
\hline 0 & $41.83 \pm 5.70$ & $43.83 \pm 8.90$ & $2.00 \pm 4.39$ \\
0.63 & $43.50 \pm 3.92$ & $49.33 \pm 3.98$ & $5.83 \pm 2.11$ \\
1.25 & $37.66 \pm 5.01$ & $42.91 \pm 5.46$ & $5.25 \pm 6.69$ \\
2.5 & $40.66 \pm 5.71$ & $43.33 \pm 6.28$ & $2.66 \pm 2.33$ \\
\hline
\end{tabular}

Media \pm error estándar

Cuadro 3. Parámetros productivos de cabras que consumieron extracto enzimático de sustrato gastado de Pleurotus ostreatus en su alimentación.

\begin{tabular}{ccccccc}
\hline \multirow{2}{*}{ Tratamiento } & \multicolumn{5}{c}{ Ganancia diaria de peso $(\mathrm{g})$} \\
\cline { 2 - 7 } & Semana 1 & Semana 2 & Semana 3 & Semana 4 & Semana 5 & Semana 6 \\
\hline 0 & $0.14 \pm 0.25^{\mathrm{a}}$ & $-0.08 \pm 0.08^{\mathrm{a}}$ & $0.32 \pm 0.09^{\mathrm{a}}$ & $0.00 \pm 0.09^{\mathrm{b}}$ & $0.28 \pm 0.10^{\mathrm{a}}$ & $0.04 \pm 0.05^{\mathrm{b}}$ \\
0.63 & $0.32 \pm 0.18^{\mathrm{a}}$ & $-0.25 \pm 0.09^{\mathrm{a}}$ & $0.26 \pm 0.07^{\mathrm{a}}$ & $0.19 \pm 0.07^{\mathrm{a}}$ & $0.14 \pm 0.10^{\mathrm{ab}}$ & $0.19 \pm 0.10^{\mathrm{a}}$ \\
1.25 & $0.35 \pm 0.29^{\mathrm{a}}$ & $-0.18 \pm 0.17^{\mathrm{a}}$ & $0.23 \pm 0.07^{\mathrm{a}}$ & $0.07 \pm 0.05^{\mathrm{ab}}$ & $0.23 \pm 0.07^{\mathrm{a}}$ & $0.06 \pm 0.11^{\mathrm{b}}$ \\
2.50 & $0.19 \pm 0.22^{\mathrm{a}}$ & $-0.35 \pm 0.53^{\mathrm{a}}$ & $0.16 \pm 0.09^{\mathrm{a}}$ & $0.10 \pm 0.04^{\mathrm{ab}}$ & $0.03 \pm 0.09^{\mathrm{b}}$ & $0.06 \pm 0.04^{\mathrm{b}}$ \\
\hline
\end{tabular}

$\mathrm{a}, \mathrm{b}=$ Literales diferentes en filas, indican diferencia significativa entre tratamientos $(\mathrm{P}<0.05)$.

Media \pm error estándar

Cuadro 4. Ganancia diaria de peso semanal de cabras que consumieron extracto enzimático de sustrato gastado de Pleurotus ostreatus en su alimentación.

En el cuadro 5 se muestra que la producción de leche de cabras en pastoreo que consumieron extracto enzimático de sustrato gastado de Pleurotus ostreatus aumentó $(P<0.05)$ en las primeras tres semanas, lo cual difiere con lo reportado por González (2002), quien encontró que la producción de leche en cabras no se ve afectada al suplementar con un complejo enzimático de xilanasas y por El-Aziz et al., (2012) quienes encontraron que al incluir enzimas fibrolíticas (fabricadas a partir de fuentes naturales de bacterias ruminales anaeróbicas, patente 22155, Molecular Biology Lab., Ain Shams University, El Cairo, Egipto) no se incrementó la producción de leche de búfalas lactantes. 
De manera contraria a lo obtenido por Kholif et al., (2015) quienes reportaron un aumento en la producción de leche en cabras que se alimentaron con preparaciones enzimáticas de Asperozym $\AA^{\circledR}$, ASP y Tomoko®, TOM.

\begin{tabular}{|c|c|c|c|c|c|c|}
\hline Tratamiento & & & Prod & leche $(L)$ & & \\
\hline 0 & $0.46 \pm 0.40^{b}$ & $0.99 \pm 0.76^{b}$ & $1.19 \pm 0.82^{b}$ & $0.72 \pm 0.69^{b}$ & $0.75 \pm 0.77^{a}$ & $0.72 \pm 0.68^{a}$ \\
\hline 0.63 & $2.17 \pm 0.57^{a}$ & $2.23 \pm 0.49^{a}$ & $2.39 \pm 0.56^{\mathrm{a}}$ & $2.29 \pm 0.79^{a}$ & $0.74 \pm 0.86^{a}$ & $0.53 \pm 0.73^{\mathrm{a}}$ \\
\hline 1.25 & $0.71 \pm 0.19^{b}$ & $1.39 \pm 0.44^{\mathrm{ab}}$ & $1.22 \pm 0.23^{\mathrm{ab}}$ & $1.43 \pm 0.63^{\mathrm{ab}}$ & $1.46 \pm 0.64^{a}$ & $1.77 \pm 0.80^{\mathrm{a}}$ \\
\hline 2.5 & $1.34 \pm 0.70^{\mathrm{ab}}$ & $0.94 \pm 0.36^{b}$ & $1.46 \pm 0.47^{\mathrm{ab}}$ & $1.83 \pm 0.48^{\mathrm{ab}}$ & $1.55 \pm 0.51^{\mathrm{a}}$ & $1.32 \pm 0.61^{\mathrm{a}}$ \\
\hline
\end{tabular}

$\mathrm{a}, \mathrm{b}=$ Literales diferentes en filas, indican diferencia significativa entre tratamientos $(\mathrm{P}<0.05)$.

Media \pm error estándar

Cuadro 5. Producción de leche de cabras que consumieron extracto enzimático de sustrato gastado de Pleurotus ostreatus en su alimentación.

El consumo (Cuadro 6) del concentrado no fue diferente $(\mathrm{P}<0.05)$ a partir de la semana cuatro, como lo indican Dean et al., (2013), quienes encontraron que al incluir enzima fibrolítica comercial Promote $\AA^{\circledR}$ en la dieta de vacas lecheras, el consumo no fue afectado; al igual que Vargas et al., (2013), quienes incluyeron diferentes niveles de enzimas exógenas Fibrozyme ${ }^{\circledR}$ en dietas de novillos. A diferencia de lo reportado por Salem et al., (2015), quienes incluyeron un coctel enzimático (ZAD1®, ZAD2® y ZADO®) sobre un arbusto forrajero Atriplex halimus, consumido por ovejas, las cuales disminuyeron su consumo, al igual que lo encontrado por Kholif et al., (2014), quienes sustituyeron Trifolium alexandrinum por paja de arroz tratada con Pleurotus ostreatus en dietas para cabras lactantes.

\begin{tabular}{|c|c|c|c|c|}
\hline \multirow{3}{*}{ Tratamiento } & \multicolumn{4}{|c|}{ Consumo de concentrado } \\
\hline & & & & \\
\hline & Semana 1 & Semana 2 & Semana 3 & Semana 4 a 6 \\
\hline 0 & $1.30 \pm 0.16^{\mathrm{b}}$ & $1.31 \pm 0.20^{\mathrm{a}}$ & $1.26 \pm 0.10^{\mathrm{b}}$ & \\
\hline 0.63 & $1.40 \pm 0.00^{\mathrm{a}}$ & $1.36 \pm 0.08^{a}$ & $1.38 \pm 0.04^{\mathrm{a}}$ & \\
\hline 1.25 & $1.31 \pm 0.16^{b}$ & $1.40 \pm 0.00^{\mathrm{a}}$ & $1.40 \pm 0.00^{\mathrm{a}}$ & $1.40 \pm 0.00^{\mathrm{a}}$ \\
\hline 2.5 & $131 \pm 0.20^{b}$ & $1.33 \pm 0.16^{a}$ & $1.36 \pm 0.08^{\mathrm{ab}}$ & \\
\hline
\end{tabular}

Cuadro 6. Consumo de concentrado con extracto enzimático de sustrato gastado de Pleurotus ostreatus, de cabras en pastoreo.

\section{CONCLUSIÓN}

La inclusión de extracto enzimático de sustrato gastado de Pleurotus ostreatus en el concentrado de cabras en pastoreo, tiene efecto positivo sobre la producción de leche y la ganancia diaria de peso. 


\section{IMPLICACIONES}

Aunque la inclusión de extracto enzimático de sustrato gastado de Pleurotus ostreatus en el concentrado ofrecido durante la ordeña de cabras en pastoreo afecta positivamente la producción láctea y la ganancia diaria de peso; es necesario utilizarlo en cabras en confinamiento para evaluar si el efecto sería el mismo.

\section{LITERATURA CITADA}

ARCE CO, Mendoza G, Miranda LA, Meneses M, Loera O. 2013a. Efficiency of Lignocellulolytic Extracts from Thermotolerant Strain Fomes sp. EUM1: Stability and Digestibility of Agricultural Wastes. Journal of Agricultural Science and Technology. 15:229 - 240.

http://jast.modares.ac.ir/pdf 4946 2275679f839ed6f8c466fd13a9b7086d.html

ARCE CO, Mendoza GD, Hernández PA, Meneses M, Torres SN, Loera O. 2013b. The Effects of a Lignocellulolytic Extract of Fomes sp. EUM1 on the Intake, Digestibility, Feed Efficiency and Growth of Lambs. Animal Nutrition and Feed Technology. 13: 363-372. Print ISSN: 0972-2963. Online ISSN: 0974-181X.

AYALA MM, González MSS, Pinos RJM, Vázquez C, Meneses MM, Loera CO, Mendoza GD. 2011. Fibrolytic potential of spent compost of the mushroom agaricus bisporus to degrade forages for ruminants. African Journal of Microbiology research. 5(3): 241-249. https://www.uaeh.edu.mx/investigacion/icap/LI NutrMinRum/Maric Ayala/1.pdf

BEAUCHEMIN KA, Rode LM, Maekawa M, Morgavi DP, Kampen R. 2000. Evaluation of a nonstarch polysaccharidase feed enzyme in dairy cow diets. Journal of Dairy Science. 83:543 - 553. DOI: http://dx.doi.org/10.3168/jds.S0022-0302(00)74914-9

BOURBONNAIS R, Paice G, Freiermuth MB, Bodie E, Borneman S. 1997. Reactivities of various mediators and lacasses with kraft pulp and lignin model compounds. Aplied Environment Microbiology. 63:4627 - 4632.

http://aem.asm.org/content/63/12/4627.full.pdf

BUENO LA, Mendoza MGD, Hernández GPA, Martínez GJA, Plata PFX. 2013. Evaluation of High Doses of Exogenous Fibrolytic Enzymes in Lambs Fed an Oat Straw Based Ration. Animal Nutrition and Feed Technology. 13: 355-362. Print ISSN: 0972-2963 Online ISSN: 0974-181X.

BRADFORD MM. 1976. A rapid and sensitive method for the quantitation of microgram quantities of protein utilizing the principle of protein-dye binding. Analytic Biochemical. 72:248 - 254. https://doi.org/10.1016/0003-2697(76)90527-3 
CAJA G, González E, Flores C, Carro MD, Albanell E. 2003. Alternativas a los antibióticos de uso alimentario en rumiantes: probióticos, enzimas y ácidos orgánicos. Sitio Argentino de Producción Animal. 18 pag. http://www.produccionanimal.com.ar/informacion tecnica/invernada promotores crecimiento/02alternativas a los antibioticos.pdf

DEAN DB, Staples CR, Littell RC, Kim S, Adesogan AT. 2013. Effect of Method of Adding a Fibrolytic Enzyme to Dairy Cow Diets on Feed Intake Digestibility, Milk Production, Ruminal Fermentation, and Blood Metabolites. Animal Nutrition and Feed Technology. 13: 337-353.

https://www.researchgate.net/publication/260001238 Effect of Method of Adding a F ibrolytic Enzyme to Dairy Cow Diets on Feed Intake Digestibility Milk Production Ruminal Fermentation and Blood Metabolites

EL-AZIZ AM, Kholif SM, Morsy TA. 2012. Buffalo's Milk Composition and Its Fat Properties as Affected by Feeding Diet Supplemented with Flaxseed or Fibrolytic Enzymes in Early Lactation. Journal of Life Sciences. 4(1):19-25. http://www.krepublishers.com/02Journals/JLS/JLS-04-0-000-12-Web/JLS-04-1-000-12-Abst-PDF/JLS-04-1-019-12-130El-Aziz-M-A/JLS-04-1-019-12-130-El-Aziz-M-A-Tt.pdf

GONZÁLEZ GE. 2002. Use of fibrolytic enzymes in dairy goats. In vitro evaluation of activity and fermentative characteristics. Tesis doctoral. Facultad de Veterinaria. Departamento de Ciencia Animal y de los Alimentos. Bellaterra, Barcelona. España. 77p.

KHOLIF AE, Khattab AM, El-Shewy AA, Salem AZM, Kholif AM, El-Sayed MM, Gado HM, Mariezcurrena MD. 2014. Nutrient Digestibility, Ruminal Fermentation Activities, Serum Parameters and Milk Production and Composition of Lactating Goats Fed Diets Containing Rice Straw Treated with Pleurotus ostreatus. Asian-Australasian Journal of Animal Sciences. 27(3):357-364. DOI: 10.5713/ajas.2013.13405

KHOLIF SM, Morsy TA, Matloup OH, Ebeid HM, El-Kader MKA. 2015. Effects of Crushed Linseed or Linseed Oil Supplementation on Performance of Dairy Goats and Fatty Acid Profile in Milk. Life Science Journal. 12(2s). DOI: 10.12816/0016602

LOERA O, Cordova J. 2003. Improvement of xylanase production by a parasexual cross between Aspergillus niger strains. Brazilian Archive Biology Technology. 46:177-181. http://www.scielo.br/pdf/babt/v46n2/16283.pdf

MÁRQUEZ AT, Mendoza MGD, González MSS, Buntinx DSE, Loera CO. 2007. Actividad fibrolítica de enzimas producidas por Trametes sp. EUM1, Pleurotus ostreatus IE8 y Aspergillus niger AD96.4 en fermentación sólida. Interciencia. 32(11):780-785. http://www.redalyc.org/pdf/339/33901110.pdf 
MILLER GM. 1959. Use of dinitrosalicylic acid reagent for determination of reducing sugar. Analytic Chemical. 31:426-428. http://pubs.acs.org/doi/pdf/10.1021/ac60147a030

SALEM AZM, Alsersy H, Camacho LM, El-Adawy MM, Elghandour MMY, Kholif AE, Rivero N, Alonso MU, Zaragoza A. 2015. Feed intake, nutrient digestibility, nitrogen utilization, and ruminal fermentation activities in sheep fed Atriplex halimus ensiled with three developed enzyme cocktails. Czech Journal of Animal Science. 60(4):185-194. http://www.agriculturejournals.cz/publicFiles/150686.pdf

SALINAS GH, Maldonado JA, Torres HG, Triana GM, Isidro RLM, Meda AP. 2015. Compositional quality of local goat milk in the Comarca Lagunera of Mexico. Revista Chapingo Serie Zonas Áridas. 14(2):175-184.

http://www.redalyc.org/articulo.oa?id=455544908001

SÁNCHEZ SP, Meneses MM, Torres SN 2015. Production of Lignocellulolytic Enzymes with Pleurotus ostreatus-IE8 by Solid Fermentation and its Effect on the Chemical Composition of Sugarcane Bagasse. Life Science Journal. 12(2s):37-41. http://www.lifesciencesite.com/lsj/life1202s15/006 A00376life1202s15 LifeSI26 3741. pdf

SIGMAPLOT. 2006. Sigmaplot. Systat Software. Ver. 10. Inc. Sigmaplot for Windows.

SPSS. 2011. Manual de usuario del sistema básico de IBM SPSS Statistics 20. IBM. USA.

TORRES N, Mendoza GD, Bárcena R, Loera O, González S, Aranda E, Hernández PA, Crosby M. 2013. Effects of Various Fibrolytic Enzyme Extracts on Digestibility and Productive Performance of Lambs Fed a Forage-Based Diet. Animal Nutrition and Feed Technology. 13: 381-389. Print ISSN: 0972-2963. Online ISSN: 0974-181X

VAN KUIJK SJA, Sonnenberg ASM, Baars JJP, Hendricks WH, Cone JW. 2015. Fungal treated lignocellulosic biomass as ruminant feed ingredient: A review. Biotechnology Advances. 33:191-202. http://dx.doi.org/10.1016/j.biotechadv.2014.10.014

VARGAS JM, Mendoza GD, Rubio LMS, Castrejón FA. 2013. Effect of Exogenous Fibrolytic Enzymes on the Carcass Characteristics and Performance of Grain-Finished Steers. Animal Nutrition and Feed Technology. 13:435-439. http://wwwnaweb.iaea.org/nafa/aph/public/EXOGENOUS-ENZYMES-in-ANIMAL-NUTRITION.pdf

\section{AGRADECIMIENTOS}

El presente trabajo fue financiado por SEP-PROMEP con número de asignación DSA/103.5/16/10281. 